\title{
Orientação Exterior de Imagens CBERS-4/PAN Utilizando Modelos Rigorosos
}

\section{Exterior Orientation of CBERS-4/PAN Imagery Using Rigorous Models}

\author{
Thales Shoiti Akiyama ${ }^{1}$, José Marcato Junior ${ }^{2}$, Antonio Maria Garcia Tommaselli ${ }^{3}$, Tiago Lima Rodrigues ${ }^{4}$ e Nayara \\ Vasconcelos Estrabis 5
}

1 Universidade Federal de Mato Grosso do Sul, Faculdade de Engenharias, Arquitetura e Urbanismo e Geografia, Campo Grande, Brasil. akiyama.thales@gmail.com.

ORCID: https://orcid.org/0000-0002-1397-3501

2 Universidade Federal de Mato Grosso do Sul, Faculdade de Engenharias, Arquitetura e Urbanismo e Geografia, Campo Grande, Brasil. jose.marcato@ufms.br.

ORCID: https://orcid.org/0000-0002-9096-6866

3 Universidade Estadual Paulista, Departamento de Cartografia, Presidente Prudente, Brasil. a.tommaselli@unesp.br. ORCID: https://orcid.org/0000-0003-0483-1103

4 Universidade Federal do Paraná, Programa de Pós-graduação em Ciências Geodésicas, Curitiba, Brasil. tiagorodrigues@ufpr.br. ORCID: https://orcid.org/0000-0002-3037-9037

5 Universidade Federal de Mato Grosso do Sul, Faculdade de Engenharias, Arquitetura e Urbanismo e Geografia, Campo Grande, Brasil.nayara.estrabis@ufms.br.

ORCID: https://orcid.org/0000-0002-5249-3893

Recebido: 08.2019| Aceito: 11.2020

Resumo: O processo de correção geométrica em imagens orbitais é uma etapa indispensável, em que é possível a geração de produto cartográfico mais confiável, o qual pode ser utilizado em inúmeras aplicações. Os satélites de imageamento possuem sensores embarcados integrados com outros dispositivos que, em conjunto, são capazes de fornecer as imagens orbitais, as quais apresentam diferentes níveis de detalhamento e de acurácia posicional. Desta forma, é importante analisar a qualidade das imagens orbitais do CBERS-4/PAN na banda pancromática, cujo GSD (Ground Sample Distance) é de 5 metros, bem como quais procedimentos podem ser adotados para melhorá-las. Além do CBERS-4 ser considerado um satélite recente, são escassos os estudos referentes à confiabilidade posicional e correção geométrica dos produtos gerados, principalmente das imagens do sensor PAN (Panchromatic and Multispectral Camera). Para a orientação exterior das imagens CBERS-4/PAN foi utilizado o modelo matemático rigoroso baseado nas equações de colinearidade adaptadas para o sensor de varredura linear. Foram utilizados dados orbitais como injunções relativas, e variou-se a quantidade de pontos de apoio - 15, 10 e 5 pontos, cujas coordenadas são oriundas dos limites de propriedades rurais cadastrados no INCRA (Instituto Nacional de Colonização e Reforma Agrária). Foram geradas imagens corrigidas geometricamente com acurácia posicional planimétrica mais confiáveis (10 a $15 \mathrm{~m})$.

Palavras-chave: Fotogrametria. Sensoriamento Remoto. Cartografia. Acurácia Posicional.

\begin{abstract}
The process of geometric correction in orbital images is an indispensable step, that makes feasible the generation of reliable cartographic products, which can be used in many applications. The imagery satellites have embedded sensors integrated with other devices that jointly provide orbital images, which present different levels of details and positional accuracy. Thus, it is important to analyze the quality of the orbital images of the CBERS-4/PAN, which presents a GSD (Ground Sample Distance) of $5 \mathrm{~m}$ as well as the procedures that can be adopted to improve them. CBERS-4 is considered a recent satellite and there are few studies on its positional reliability and geometric correction of generated products, mainly of PAN (Panchromatic and Multispectral Camera) images. In this work, the exterior orientation of the CBERS-4/PAN images was performed with the rigorous mathematical model based on the collinearity equations adapted for the pushbroom sensor model. Orbital data were used as a relative constraints and the number of control points were varied - 15, 10 and 5, which coordinates were obtained from the land parcel boundaries registered in INCRA (National Institute of Colonization and Agrarian Reform). Geometrically corrected images with more reliable positional accuracy $(10$ to $15 \mathrm{~m}$ ) were generated.

Keywords: Photogrammetry. Remote Sensing. Cartography. Positional Accuracy.
\end{abstract}




\section{INTRODUÇÃO}

As imagens obtidas por sensores orbitais podem ser utilizadas em diversas áreas, tais como: vegetação, identificação de queimadas, agricultura, identificação de atividades humanas ao longo de cursos d'água, estradas, mapeamento de uso do solo, monitoramento de reservatórios, cartografia, entre outros (FONSECA et al., 2014; INPE, 2019). Em 1988, iniciou-se o programa espacial CBERS (China-Brazil Earth Resources Satellite), advindo de uma parceria técnico-científica entre o Brasil e a China. Este programa é identificado como uma potencial fonte de dados para o monitoramento de dinâmicas da superfície terrestre. Suas imagens são fornecidas gratuitamente pelo Instituto Nacional de Pesquisas Espaciais (INPE) e disponíveis como suporte para pesquisas científicas. O CBERS-4 é um dos diversos satélites que já foram produzidos pelo programa, e no seu módulo carga útil são utilizadas quatro câmeras: Câmera Multiespectral Regular - MUX (Multispectral Camera); Câmera de Campo Largo - WFI (Wide-Field Imaging Camera); Câmera Pancromática e Multiespectral - PAN (Panchromatic and Multispectral Camera); e Imageador Multiespectral e Termal - IRS (Infrared Medium Resolution Scanner). Essas câmeras possuem desempenhos radiométricos e geométricos melhorados em relação aos satélites que foram lançados anteriormente.

Por que é importante orientar as imagens obtidas por sensores remotos e corrigi-las geometricamente? Geralmente tais imagens apresentam distorções radiométricas e geométricas significantes, e devido a isso, não podem ser utilizadas diretamente dependendo do tipo de aplicação desejada. Um exemplo de estudo que mostra a importância da correção geométrica é o de Devaraj e Shah (2014), que procuraram corrigir as imagens do sensor HRC (High Resolution CCD Camera) de GSD de $20 \mathrm{~m}$ a bordo dos satélites CBERS-2 e CBERS-2B. Inicialmente, as imagens apresentaram acurácia posicional entre 2 e $11 \mathrm{~km}$, e após as técnicas de correção geométrica, as imagens dos satélites CBERS-2 e CBERS-2B apresentaram uma acurácia posicional - expressa pelo REQM (Raiz do Erro Quadrático Médio) de 17,04 m e 16,34 m, respectivamente.

Desta forma, para serem gerados produtos cartográficos confiáveis, são necessários procedimentos para a orientação e posterior correção geométrica das imagens orbitais. Para orientar a imagem com relação a um Sistema de Coordenadas do espaço objeto e minimizar as distorções geométricas, são utilizadas funções e modelos matemáticos no processo de correção geométrica de tais imagens. Dentre os modelos existentes, são classificados em generalizados e rigorosos, onde este último é dividido em dois tipos: modelo Posição-Rotação e Órbita-Atitude. Todos estes procedimentos para garantir a qualidade geométrica são essenciais para validar a relação entre pontos no terreno e suas respectivas posições nas imagens.

O CBERS-4 foi lançado em 07 de dezembro de 2014 e foram poucos os estudos realizados em suas imagens considerando as questões de confiabilidade posicional e procedimentos de correção geométrica. No Brasil, houve alguns estudos que avaliaram a questão da confiabilidade posicional utilizando as imagens do CBERS-4, como o de Statella (2016), que analisou a acurácia posicional de imagens do sensor MUX, a qual apresentou deslocamento posicional de aproximadamente $404 \mathrm{~m}$. Para a correção geométrica, o autor utilizou as transformações polinomiais de ordem 1 e 2, gerando REQM de 39,20 m para o polinômio de ordem 1 e 34,30 m para o polinômio de ordem 2; Marcato Junior et al. (2017) avaliaram a acurácia posicional de imagens do sensor PAN, e verificaram um erro planimétrico de 614 m; e o de Akiyama, Marcato Junior e Tommaselli (2018) que utilizaram modelos matemáticos generalizados (não físicos) para a correção geométrica de imagens CBERS-4/PAN.

Assim sendo, o objetivo deste trabalho é o de investigar a orientação exterior de imagens do CBERS4/PAN utilizando o modelo matemático rigoroso do tipo Órbita-Atitude. pois são escassos os estudos que utilizam este modelo para as imagens obtidas a partir deste sensor.

\section{IMAGENS ORBITAIS DOS SATÉLITES CBERTS-4/PAN}

As imagens CBERS-4/PAN disponibilizadas pelo INPE possuem diversos níveis de processamento, conforme descritos na Tabela 1, no qual as imagens de nível 0 e 1 apenas são fornecidas a usuários mediante solicitação, enquanto as de nível 2, 3 e 4 podem ser obtidas gratuitamente. Quanto maior o grau do nível de processamento, mais processos de correção são aplicados para gerar um produto de melhor qualidade. $\mathrm{O}$ 
processo de correção geométrica a partir do chamado georreferenciamento direto (sem pontos de apoio) depende unicamente de dados orbitais (posição e velocidade do satélite), de informações sobre a montagem do instrumento no satélite (ângulos de boresight e deslocamentos entre o Centro Perspectivo (CP) e o centro de fase da antena do sistema de posicionamento por satélite que fornece a posição), da geometria de visada do instrumento (Parâmetros de Orientação Interior (POI) da câmera) e de um modelo representativo da superfície terrestre (elipsóide de revolução). Ao analisar as imagens de nível 4 (ortorretificadas com pontos de apoio), disponibilizadas pelo INPE, verificou-se, a partir dos arquivos XML (Extensible MarkUp Language), resíduos em pontos de apoio usados na correção geométrica acima de 11 GSD $(55 \mathrm{~m})$.

Tabela 1 - Níveis de processamento das imagens CBERS.

\begin{tabular}{|c|c|}
\hline Sensor & Descrição \\
\hline Nível 0 & $\begin{array}{c}\text { Imagem recebida diretamente pela estação de recepção do INPE (imagem em estado } \\
\text { bruto). }\end{array}$ \\
\hline Nível 1 & $\begin{array}{l}\text { Imagem do nível } 0 \text { com correção radiométrica (calibração e restauração do sinal } \\
\text { transmitido). }\end{array}$ \\
\hline Nível 2 & $\begin{array}{c}\text { Imagem do nível } 1 \text { com correção geométrica, aplicada com o uso de dados orbitais de } \\
\text { SCOA (Sistemas de Controle e Orbita de Atitude), de informações sobre a montagem do } \\
\text { instrumento no satélite, da geometria de visada do instrumento e de um modelo } \\
\text { representativo da superfície terrestre. }\end{array}$ \\
\hline Nível 3 & $\begin{array}{c}\text { Imagem do nível } 2 \text { aplicados procedimentos adicionais de correção geométrica com o } \\
\text { uso de pontos de apoio. }\end{array}$ \\
\hline Nível 4 & $\begin{array}{l}\text { Imagem do nível } 2 \text { ortorretificada com o uso de um MDT (Modelo Digital de Terreno) } \\
\text { ou um MNET (Modelo Numérico de Elevação do Terreno). }\end{array}$ \\
\hline
\end{tabular}

Fonte: Machado e Silva (2007).

Foi utilizada para este estudo uma imagem CBERS-4/PAN de nível 1, pois não possuem procedimentos de correção geométrica previamente aplicados, coletada no dia 02 de julho de 2017, correspondente à órbita 165 e ponto 123. Seu arquivo XML contém informações (valores dos ângulos de atitude roll, pitch e yaw, velocidades da plataforma $V x$, $V y$ e $V z$ para um determinado instante de coleta, matriz dos ângulos de boresight em forma de quatérnions, número de colunas e linhas da imagem, tamanho do pixel) importantes para a utilização do modelo rigoroso. A Tabela 2 mostra os POI e os ângulos de boresight do sensor PAN.

Tabela 2 - Características técnicas do sensor PAN.

\begin{tabular}{c|c|c}
\hline \multicolumn{2}{c}{ Distância focal $(\mathbf{m m})$} & $1010 \mathrm{~mm}$ \\
\hline \multicolumn{2}{c}{ Resolução Espacial $(\mathbf{m})$} & $5 \mathrm{~m}$ (Banda pancromática) \\
\hline \multicolumn{2}{c}{ Tamanho do detector $(\mathbf{m m})$} & $0,0065 \mathrm{~mm}$. \\
\hline \multicolumn{2}{c}{ Número de colunas $($ pixels) } & 12000 \\
\hline \multirow{2}{*}{$\begin{array}{c}\text { Ângulos de } \\
\text { boresight }\end{array}$} & $\boldsymbol{b x}^{\mathbf{0}}$ & 0,400973794 \\
\cline { 2 - 3 } & $\boldsymbol{b y}^{\mathbf{0}}$ & 0,172116780 \\
\cline { 2 - 3 } & $\boldsymbol{b z}^{\mathbf{0}}$ & 0,056603128 \\
\hline
\end{tabular}

Fonte: INPE (2019).

\section{MATERIAIS E MÉTODOS}

Jacobsen (2005) menciona que os satélites de alta resolução espacial são equipados com SCOA, sensores de orientação direta (GNSS, giroscópios, sensores de estrelas, dentre outros), e outros equipamentos. Entretanto, mesmo que essas imagens sejam corrigidas a partir de orientação direta, sem pontos de controle, e utilizando informações intrínsecas dos sensores, nem sempre atingem a acurácia requerida para determinadas aplicações. À vista disso, é necessário utilizar feições de controle (ponto, reta ou área) em conjunto com determinados modelos matemáticos para orientação das imagens. Existem dois tipos de modelos que visam corrigir geometricamente as imagens e assim melhorar a acurácia posicional delas: os modelos generalizados (KIM; DOWMAN, 2006), também denominados empíricos (TOUTIN, 2004) ou genéricos (KOCAMAN, 2008), e os modelos rigorosos (ou físicos). 
O modelo generalizado procura relacionar o espaço objeto (sistema de referência terrestre) e o espaço imagem utilizando feições de apoio e não se tem conhecimento das informações dos parâmetros de aquisição do sistema. Já o modelo rigoroso procura descrever a geometria de aquisição da cena utilizando feições de apoio e as informações do modelo sensor e da plataforma. Jacobsen, Büyüksalih e Topan (2005) mencionam que o georreferenciamento direto pode ser acurado, mas geralmente para uma melhora ou validação do produto é requerida a utilização destes pontos por conta da limitação na qualidade dos dados obtidos em órbita e em pequena parte também pela alteração dos valores nominais dos POI e dos parâmetros de montagem.

No presente trabalho utilizaram-se as equações de colinearidade (Seção 3.1), que procura mostrar o modelo rigoroso adotado para o sensor de varredura linear. As configurações dos pontos de apoio e verificação são apresentadas na Seção 3.2. Na Seção 3.3 apresenta-se o procedimento adotado para a validação da correção geométrica.

\subsection{Equações de colinearidade}

O modelo matemático rigoroso que relaciona o espaço objeto com o espaço imagem é baseado na equação de colinearidade, que se objetiva por modelar o princípio de colinearidade no instante de tomada da imagem entre um ponto no espaço objeto, o seu homólogo no espaço imagem e o CP (Centro Perspectivo) (BOGGIONE, 2014; MIKHAIL; BETHEL; MCGLONE, 2001; MARCATO JUNIOR; TOMMASELLI, 2013). Esta equação exige o conhecimento de dados de calibração do sensor, informações da órbita do satélite, geometria de aquisição da imagem e atitude do sensor.

A seguir é apresentada a Eq. (1), que representa matricialmente as equações de colinearidade adaptadas para a geometria de varredura linear, considerando dados de órbita - Modelo Órbita-Atitude (KIM; DOWMAN, 2006). Este modelo foi implementado no programa TMS (Triangulação MultiSsensor) (MARCATO JUNIOR, 2011).

$$
\left[\begin{array}{c}
x_{S} \\
y_{S} \\
-f
\end{array}\right]=\left[\begin{array}{c}
0 \\
y_{S} \\
-f
\end{array}\right]=\lambda\left[R_{S}^{P}\right]^{T}\left[R_{P}^{O}\right]^{T}\left[R_{O}^{T}\right]^{T}\left[\begin{array}{c}
X-X_{S} \\
Y-Y_{S} \\
Z-Z_{S}
\end{array}\right]
$$

O termo $R_{S}^{P}$ é a matriz de rotação dos ângulos de boresight ( $\left.b x, b y, b z\right)$, transformando do sistema de referência do sensor para o sistema de referência da plataforma, representada a seguir pela Eq. (2). Os termos $s b$ e $c b$ significam seno e cosseno dos ângulos de boresight, respectivamente.

$$
R_{S}^{P}=\left[\begin{array}{ccc}
c b_{Z} c b_{Y} & c b_{Z} s b_{Y} s b_{X}+s b_{Z} c b_{X} & -c b_{Z} s b_{Y} c b_{X}+s b_{Z} s b_{X} \\
-s b_{Z} c b_{Y} & -s b_{Z} s b_{Y} s b_{X}+c b_{Z} c b_{X} & s b_{Z} s b_{Y} c b_{X}+c b_{Z} s b_{X} \\
s b_{Y} & -c b_{Y} s b_{X} & c b_{Y} c b_{X}
\end{array}\right]
$$

$R_{P}^{O}$ (Eq. (3)) representa a matriz de rotação que relaciona o sistema de referência da plataforma e o sistema de referência orbital em função dos ângulos de atitude $\operatorname{Roll}(R)$, Pitch $(P)$ e $\operatorname{Yaw}_{(}(\psi)$ e $R_{O}^{T}$ (Eq. (4)) é a matriz de rotação que transforma do sistema orbital para o sistema de referência terrestre, como função da posição do satélite $(X s, Y s, Z s)$ e velocidade $(V x, V y, V z)$ :

$$
R_{P}^{O}=\left[\begin{array}{ccc}
\cos \psi \cos P & \cos \psi \sin P \sin R+\sin \psi \cos R & -\cos \psi \sin P \cos R+\sin \psi \sin R \\
-\sin \psi \cos P & -\sin \psi \sin P \sin R+\cos \psi \cos R & \operatorname{sen} \psi \sin P \cos R+\cos \psi \sin R \\
\sin P & -\cos P \sin R & \cos P \cos R
\end{array}\right]
$$




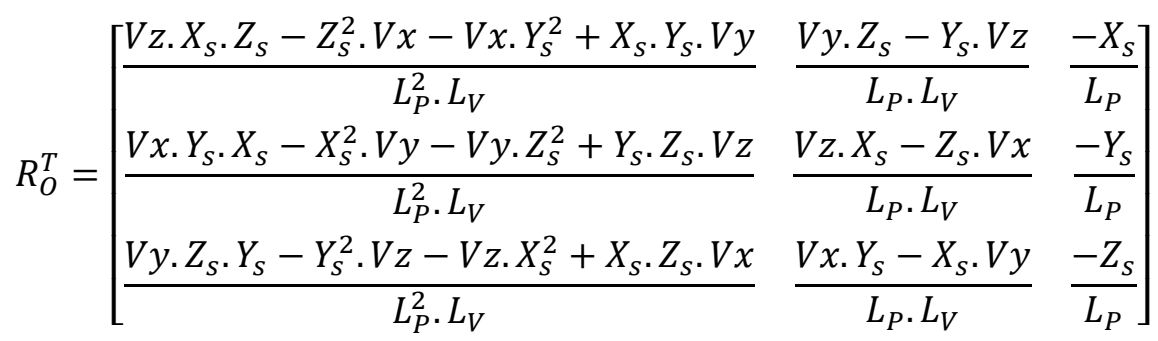

em que $L_{p}=\sqrt{\left(X_{s}^{2}+Y_{s}^{2}+Z_{s}^{2}\right)}$ e $L_{V}=\sqrt{\left(V x^{2}+V y^{2}+V z^{2}\right)}$.

Para a modelagem das alterações da posição, velocidade e ângulos de atitude do satélite durante o tempo de formação da cena, é necessário o uso de um modelo de plataforma. A seguir é apresentado o modelo de plataforma polinomial (Eq. (5)) utilizado por Kim e Dowman (2006) e Marcato Junior e Tommaselli (2013). Este modelo contém 27 parâmetros a serem determinados:

$$
\begin{gathered}
X_{S}=X_{0}+a_{1} t+b_{1} t^{2} \\
Y_{S}=Y_{0}+a_{2} t+b_{2} t^{2} \\
Z_{S}=Z_{0}+a_{3} t+b_{3} t^{2} \\
V_{X}=V_{X 0}+a_{4} t+b_{4} t^{2} \\
V_{Y}=V_{Y 0}+a_{5} t+b_{5} t^{2} \\
V_{Z}=V_{Z 0}+a_{6} t+b_{6} t^{2} \\
R=R_{0}+a_{7} t+b_{7} t^{2} \\
P=P_{0}+a_{8} t+b_{8} t^{2} \\
\psi=\psi_{0}+a_{9} t+b_{9} t^{2}
\end{gathered}
$$

em que $t$ é o tempo. Os parâmetros da Eq. (5) descrevem a trajetória e a atitude do satélite no intervalo de aquisição da imagem, e para estimar tais parâmetros foi utilizado o método combinado de ajustamento com injunção de peso.

\subsection{Orientação indireta das imagens CBERS-4/PAN}

Foram utilizados pontos de apoio para orientar indiretamente as imagens CBERS-4/PAN e para corrigir os parâmetros que definem a órbita do sensor. Estes pontos são divididos em:

a) apoio (utilizados para ajustar o modelo);

b) verificação (utilizados para validar o modelo).

Para a obtenção das coordenadas planimétricas no Sistema Geodésico Local (X, Y) de referência, foram utilizados os limites das propriedades rurais georreferenciadas por todo o Brasil disponibilizados pelo INCRA - Instituto de Colonização e Reforma Agrária (2018). No estudo de Akiyama, Marcato Junior e Tommaselli (2018), foi observado que tais limites apresentam acurácia posicional maior que $50 \mathrm{~cm}$, além de suas feições se associarem perfeitamente em relação às imagens do satélite RapidEye, também utilizadas como referência. Em relação às coordenadas altimétricas (Z) utilizadas, Marini et al. (2017) mostra que o SRTM (Shuttle Radar Topography Mission) é o que apresenta melhor acurácia de posicionamento em Mato Grosso 
do Sul, que engloba a imagem utilizada nesse trabalho. Portanto, devido a tal estudo, foram utilizados os SRTM versão 04 obtidas no site do Earth Explorer (2019).

Para a correção geométrica das imagens CBERS-4/PAN foram utilizadas como referência imagens ortorretificadas do satélite RapidEye, pois estudos realizados por Marcato Junior et al. (2015) mostraram que os valores de REQM da resultante planimétrica das imagens RapidEye foram de 1 GSD (6,5 m), concluindo que tais imagens apresentam acurácia posicional que permitem seu uso como referência para este estudo. Tais imagens são disponibilizadas no GeoCatálogo do Ministério do Meio Ambiente (MMA, 2018).

Figura 1 - Medição dos pontos de apoio correspondentes aos limites das propriedades rurais georreferenciadas associadas às imagens do satélite RapidEye. (A) Todas as imagens utilizadas; (B) Ampliação de uma parte para melhor

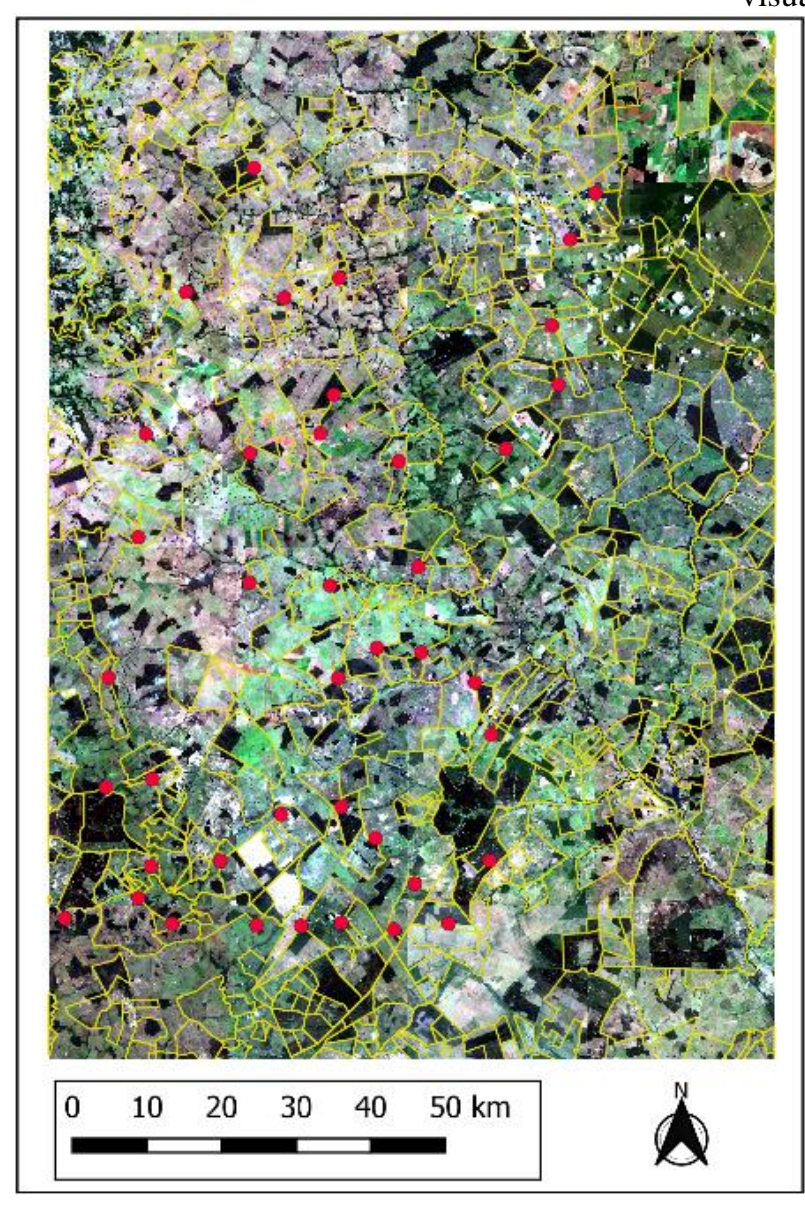

(A) visualização.

Fonte: Os autores (2021).

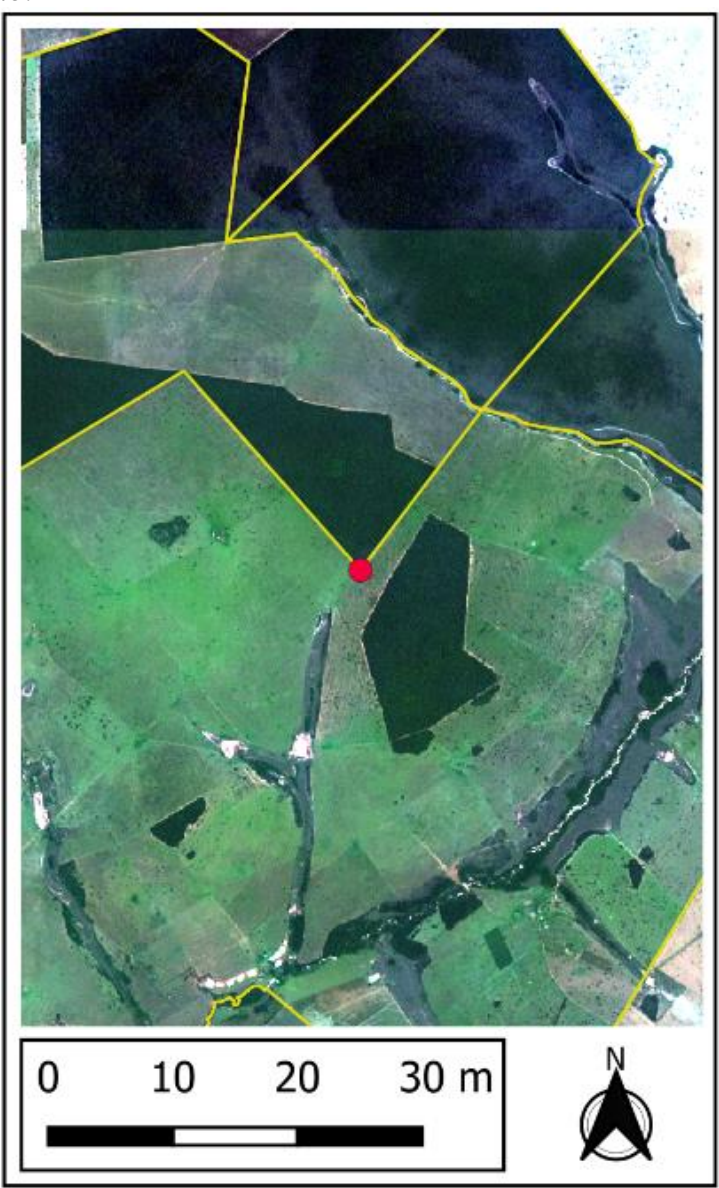

(B)

Como o modelo rigoroso descreve a geometria e aquisição da cena a partir de informações do modelo da plataforma e do sensor, ele não necessita de uma grande quantidade de pontos distribuídos sobre a imagem, assim como apresentam uma maior flexibilidade na sua distribuição em toda a imagem. Jacobsen (2007) menciona que o modelo rigoroso proporciona melhores resultados quando comparados aos modelos generalizados.

Foram utilizados 15 (quinze) pontos de apoio e 14 (quatorze) de verificação e, com o intuito de verificar se o número de pontos de apoio utilizados iria influenciar nos resultados, foram diminuindo-se tais pontos de 5 (cinco) em 5 (ver Figura 2). Marcato Junior e Tommaselli (2013) realizaram experimentos com sensor do satélite CBERS-2B usando 50, 15 e 5 pontos de apoio. Os autores verificaram que ao usar 50 ou 15 pontos de apoio não houve alteração nos resultados ao considerar as informações de órbita. Além de 15 e 5 pontos de apoio, como adotado pelos autores, realizou-se também experimento com 10 pontos de apoio. 
Figura 2 - Distribuição dos pontos de apoio (vermelho) e verificação (amarelo) na imagem 165_123 de nível 1 do CBERS-4/PAN. (A) 15 Pontos de Apoio; (B) 10 Pontos de Apoio; (C) 5 Pontos de Apoio.

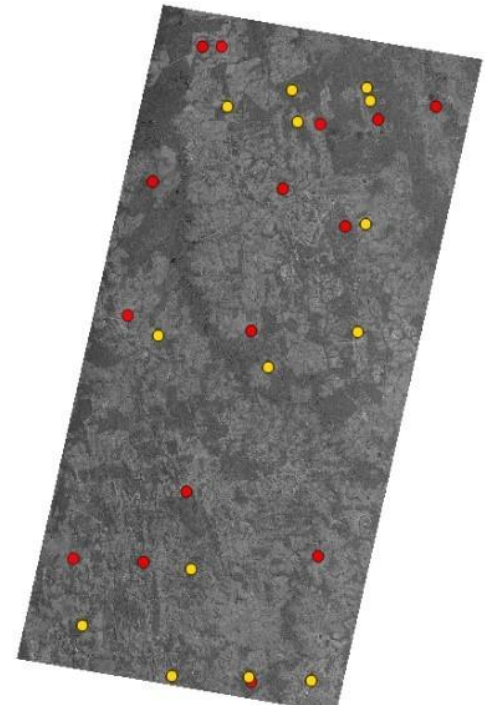

(A)

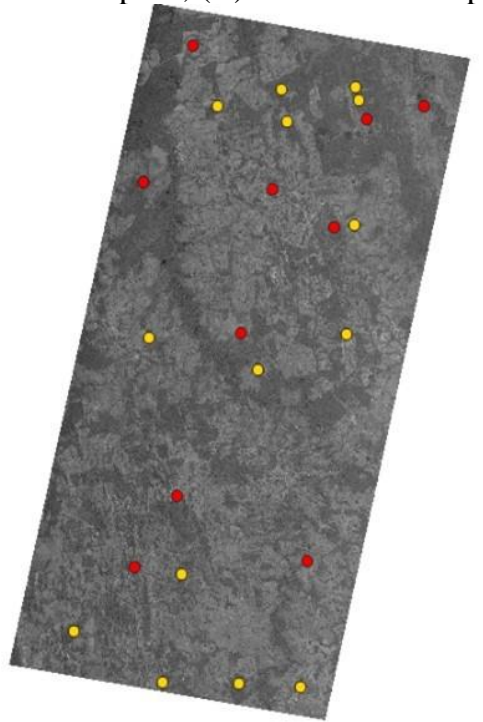

(B)

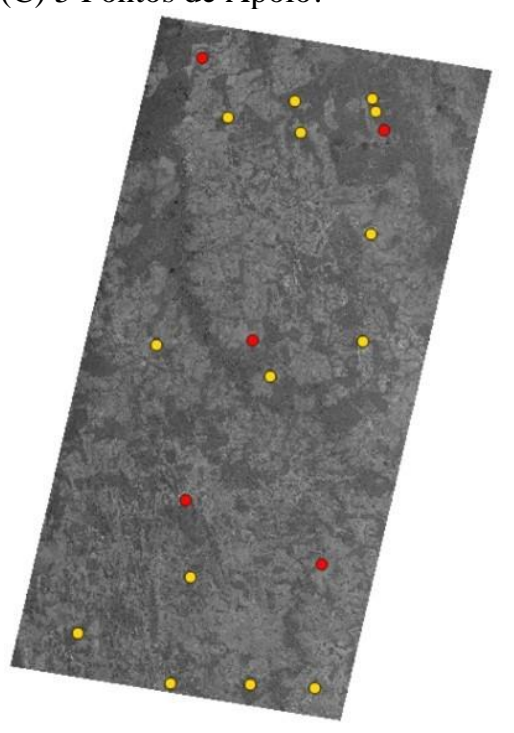

(C)

Fonte: Os autores (2021).

\subsection{Validação da correção geométrica}

Para avaliar a orientação da imagem utilizando o modelo matemático exposto são necessárias algumas etapas. Primeiramente, calcula-se a diferença entre as coordenadas dos pontos de verificação (referência) e as coordenadas destes mesmos pontos corrigidas (ajustadas). Essas diferenças podem ser consideradas como deslocamentos nas direções X e Y, calculados para cada ponto pelas seguintes formulações:

$$
\begin{gathered}
\Delta X=X_{\text {ajustado }}-X_{\text {referência }} \\
\Delta Y=Y_{\text {ajustado }}-Y_{\text {referência }}
\end{gathered}
$$

em que $\mathrm{X}_{\text {ajustado }}$ e $\mathrm{Y}_{\text {ajustado }}$ são as coordenadas dos pontos da imagem corrigidas com os modelos rigorosos e $\mathrm{X}_{\text {referencia }}$ e $\mathrm{Y}_{\text {referência }}$ são as coordenadas dos pontos de verificação. Para um conjunto de $n$ pontos, os deslocamentos resultantes nas coordenadas $\mathrm{X}$ e $\mathrm{Y}$ são definidos por:

$$
\begin{aligned}
& R E Q M \bar{X}=\sqrt{\frac{1}{n}} \sum_{i=1}^{n} \Delta X_{i}^{2} \\
& R E Q M \bar{Y}=\sqrt{\frac{1}{n}} \sum_{i=1}^{n} \Delta Y_{i}^{2}
\end{aligned}
$$

e dessa forma, pode-se calcular a REQM planimétrica (Eq. (10)), que caracteriza a acurácia de posicionamento da orientação ou possível imagem corrigida.

$$
R E Q M \text { planimétrica }=\sqrt{\left(R E Q M \bar{X}^{2}+R E Q M \bar{Y}^{2}\right)}
$$

\section{RESULTADOS E DISCUSSÃO}

A Figura 3 apresenta a REMQ planimétrica ao considerar diferentes configurações de pontos de apoio. 
Ao analisar os resultados, verifica-se que, ao reduzir o número de pontos de apoio, há um aumento nos valores. Os resultados obtidos foram menores do que $3 \mathrm{GSD}(15 \mathrm{~m})$ para todas as configurações (Tabela 3 ).

Tabela 3 - Níveis de processamento das imagens CBERS.

\begin{tabular}{l|c|c|c}
\hline Pontos de Apoio (PA) & REQM $\overline{\boldsymbol{X}}$ (GSD) & REQM $\overline{\boldsymbol{Y}}$ (GSD) & REQM planimétrica (GSD) \\
\hline $15 \mathrm{PA}$ & 1,89868 & 1,16036 & 2,22518 \\
\hline $10 \mathrm{PA}$ & 2,043259 & 1,34935 & 2,44860 \\
\hline $5 \mathrm{PA}$ & 1,847617 & 2,35948 & 2,99681 \\
\hline
\end{tabular}

Fonte: Os autores (2021).

Figura 3 - Valores de REQM variando os pontos de apoio para a imagem 165_123. (PA - Pontos de Apoio).

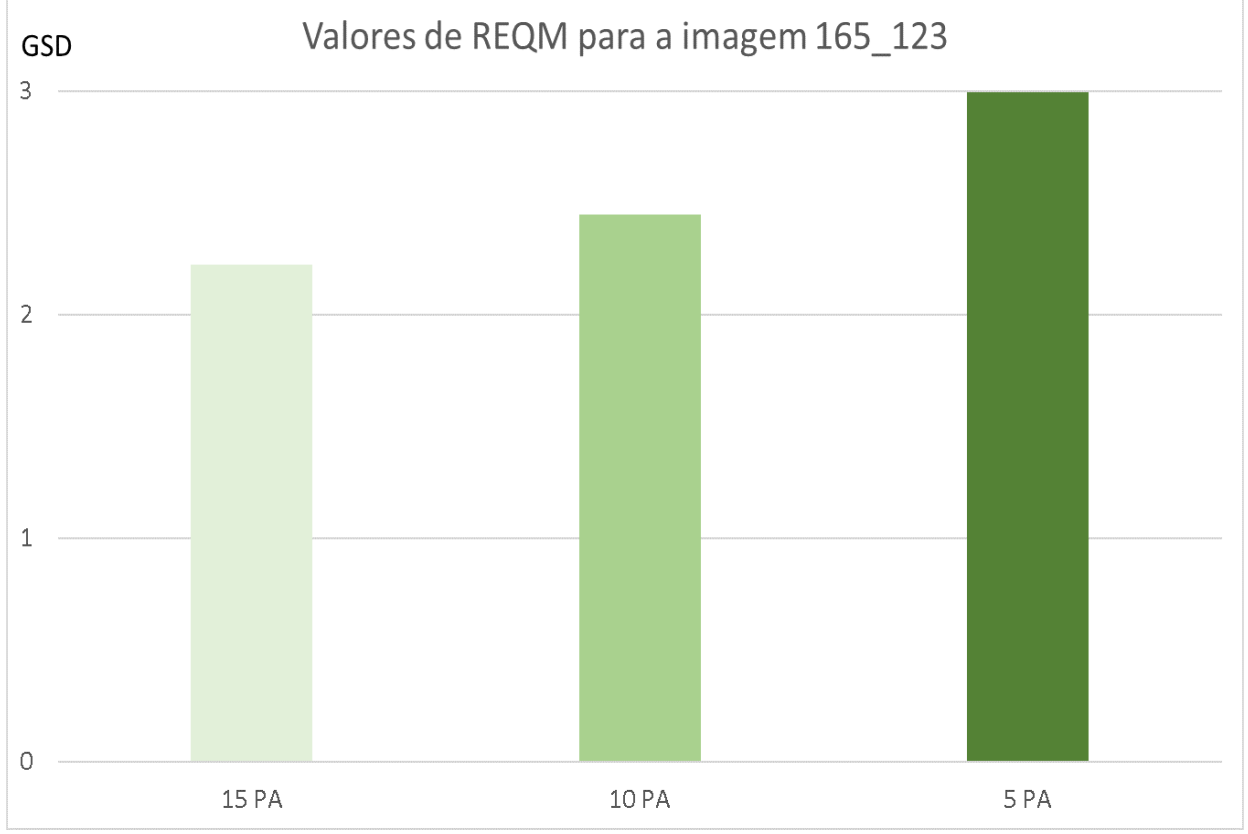

Fonte: Os autores (2021).

A qualidade das informações do sensor e da plataforma são fatores relevantes, pois quanto melhores forem os dados utilizados, melhores os resultados obtidos, e menor a quantidade de pontos de apoio necessários. No presente trabalho, realizou-se apenas o refinamento dos dados de órbita (plataforma), que gerou uma melhora significativa na acurácia posicional das imagens, conforme apresentado na Tabela 3 e Figura 3.

Alguns estudos citados anteriormente (MARCATO JUNIOR et al., 2017; AKIYAMA; MARCATO JUNIOR; TOMMASELLI, 2018) mostraram que o deslocamento posicional das imagens CBERS-4/PAN é elevado e, desta forma, o modelo rigoroso é uma alternativa para a correção geométrica e, consequentemente, obtenção de melhores resultados.

\section{CONCLUSÃO}

Este trabalho mostra a importância da aplicação correta de procedimentos para a correção geométrica de imagens CBERS-4/PAN, além de expor a eficiência do modelo matemático rigoroso utilizado. A metodologia deste trabalho surge como uma alternativa para que se possa utilizar tais imagens em inúmeras aplicações, e assim se ter um melhor aproveitamento das imagens que a câmera PAN do satélite CBERS-4 pode nos oferecer.

O trabalho não necessitou de levantamento de campo e a maioria dos materiais utilizados são gratuitos, sendo assim um estudo considerado de baixo custo, mas com uma metodologia robusta e capaz de solucionar diversos problemas referentes a questão de correção geométrica de imagens orbitais. As técnicas utilizadas neste trabalho também podem ser adaptadas para diferentes satélites, e deste modo surge como opção para buscar a aplicação de uma melhor alternativa que possa ser utilizada igualmente em todos os sensores orbitais 
que forneçam imagens de nível 1 de processamento (sem correções geométricas prévias) e dados orbitais, de montagem do sensor e alguns POI.

Akiyama, Marcato Junior e Tommaselli (2018) verificaram que os resultados da correção geométrica podem variar quando se faz o estudo em várias imagens. Devido a isto, para melhor validação da correção geométrica utilizando o modelo rigoroso, um fator que deve ser levado em conta é o estudo em mais de uma imagem para a verificação. Além disso, podem ser elaborados outros estudos nos quais pode-se analisar qual o melhor modelo matemático (rigoroso ou generalizado) a ser aplicado. Surge como uma alternativa de estudo o modelo de plataforma UCL Kepler adaptado para o modelo Órbita-Atitude, desenvolvido por Rodrigues e Machado (2019).

Os pontos de apoio foram extraídos pela interpretação do observador, com base em reconhecimento de padrões e nas características do objeto, procurando-se identificar na imagem o melhor local a posicionar essas feições de interesse. Para conseguir uma melhor obtenção destes pontos de apoio e minimizar os erros de posicionamento, uma sugestão seria o desenvolvimento de métodos ou técnicas de automação para adquirir tais pontos. Isto favoreceria tanto a redução de dependência do operador, como o aumento da precisão para o nível sub-pixel.

\section{Agradecimentos}

Os autores agradecem o suporte da FUNDECT (Fundação de Apoio ao Desenvolvimento do Ensino, Ciência e Tecnologia do Estado de Mato Grosso do Sul), ao CNPq (Conselho Nacional de Desenvolvimento Científico e Tecnológico) (p. 456149/2014-7, p.433783/2018-4 e p. 59/300.066/2015) pelo financiamento da pesquisa, o apoio da CAPES - Brasil (Coordenação de Aperfeiçoamento Pessoal de Nível Superior) - Código de Financiamento 001, e da Universidade Federal de Mato Grosso do Sul.

\section{Contribuição dos Autores}

Os autores José Marcato Junior e Antonio Maria Garcia Tommaselli contribuíram em todos os itens, desde a Conceptualização até a Redação - redação e revisão. Thales Shoiti Akiyama contribuiu na Visualização, Redação - minuta inicial e na Redação - revisão e edição. Tiago Lima Rodrigues contribuiu na Conceptualização e Redação - revisão. Nayara Vasconcelos Estrabis na Curadoria dos dados, Investigação, e Redação - revisão e edição.

\section{Conflitos de Interesse}

Os autores declaram que não há conflitos de interesse.

\section{Referências}

AKIYAMA, T. S.; MARCATO JUNIOR, J.; TOMMASELLI, A. M. G. Correção geométrica de imagens CBERS-4/PAN com modelos generalizados usando como referência dados do Sistema Nacional de Gestão Fundiária. Anuário do Instituto de Geociências - UFRJ, v.41, n. 2, p. 358-268, 2018.

BOGGIONE, G.A. Avaliação da resolução espacial de sensores ópticos orbitais. 2014. 131 p. Tese (Doutorado em Sensoriamento Remoto). Instituto Nacional de Pesquisas Espaciais, Curso de PósGraduação em Sensoriamento Remoto, São José dos Campos, 2014.

DEVARAJ, C.; SHAH, C. A. Automated geometric correction of multispectral images from High Resolution CCD Camera (HRCC) on-board CBERS-2 and CBERS-2B. ISPRS Journal of Photogrammetry and Remote Sensing, v. 89, p. 13-24, 2014.

FONSECA, L. M. G.; EPIPHANIO, J. C. N.; VALERIANO, D. M.; SOARES, J. V.; D'ALGE, J. C. L.; ALVARENGA, M. A. Earth observation applications in Brazil with focus on the CBERS program. IEEE 
Geoscience and Remote Sensing Magazine, v. 2, n. 2, p. 53-55, 2014.

INSTITUTO NACIONAL DE COLONIZAÇÃO E REFORMA AGRÁRIA (INCRA). Acervo Fundiário. Disponível em: <http:// http://acervofundiario.incra.gov.br/acervo/acv.php〉. Acesso em: 10 out. 2017.

INSTITUTO NACIONAL DE PESQUISAS ESPACIAIS (INPE). CBERS. Disponível em: <www.cbers.inpe.br>. Acesso em: 15 mai. 2019.

JACOBSEN, K.; BÜYÜKSALIH, G.; TOPAN, H. Geometric models for the orientation of high-resolution optical satellite sensors. International Archives of the Photogrammetry, Remote Sensing and Spatial Information Sciences, v. 36, 2005.

JACOBSEN, K. Orientation of high-resolution optical space images. ASPRS 2007 Annual Conference, Tampa, Florida, 2007.

KIM, T.; DOWMAN, I. Comparison of two physical sensor models for satellite images: position-rotation model and orbit-attitude model. The Photogrammetric Record, v. 21, n. 114, p. 110-123, 2006.

KOCAMAN, S.A. Sensor modeling and validation for linear array aerial and satellite imagery. 2008. 166 p. Tese (Doutorado) - Technische Wissenschaften ETH Zurich, Zurich, 2008.

MACHADO E SILVA, A. J. F. Geometria de imagens: do projeto do satélite à geração dos produtos. 2007. 224 p. Tese (Doutorado). Curso de Pós-Graduação em Sensoriamento Remoto, Instituto Nacional de Pesquisas Espaciais, São José dos Campos, 2007.

MARCATO JUNIOR, J. Fototriangulação em bloco de imagens orbitais com modelos rigorosos baseados em pontos e retas. 2011. 119 p. Dissertação (Mestrado em Ciências Cartográficas) - Programa de PósGraduação em Ciências Cartográficas, Faculdade de Ciências e Tecnologias - Universidade Estadual Paulista, Presidente Prudente, 2011.

MARCATO JUNIOR, J.; TOMMASELLI, A. M. G. Exterior orientation of CBERS-2B imagery using multifeature control and orbital data. ISPRS Journal of Photogrammetry and Remote Sensing, v. 79, p. 219$225,2013$.

MARCATO JUNIOR, J.; MARTINS, E. F. O.; MARCATO, D.; MARINI, L. B.; PARANHOS FILHO, A. C. Avaliação da qualidade geométrica de ortoimagens RapidEye disponibilizadas pelo GeoCatálogo do MMA. In: Simpósio Brasileiro de Sensoriamento Remoto (SBSR), XVII, 2015, João Pessoa. Anais..., p. 5321-5325, João Pessoa, 2015.

MARCATO JUNIOR, J.; COENIO, D. F.; GODOI, D. F.; TOMMASELLI, A. M. G.; RAMOS, A. P. M. Avaliação da acurácia posicional de imagens CBERS-4/PAN. In: Simpósio Brasileiro de Sensoriamento Remoto (SBSR), XVII, 2015, João Pessoa. Anais..., p. 5356-5361, Santos, 2017.

MARINI, L.B.; MARCATO JUNIOR, J.; RAMOS, A. P. M.; PARANHOS FILHO, A. C.; BARROS, W. M.; HIGA, L. T. Análise da acurácia altimétrica dos modelos digitais de superfície SRTM, Aster e Topodata e aplicação na representação 3D do Pantanal da Nhecolândia. Anuário do Instituto de Geociências UFRJ, v. 40, n.3, p. 48-54, 2017.

MIKHAIL, E.M.; BETHEL, J.S.; MCGLONE, J.C. Introduction to Modern Photogrammetry. New York, 2001.

MINISTÉRIO DO MEIO AMBIENTE (MMA). Geo Catálogo MMA. Disponível em: <http://geocatalogo.mma.gov.br/> Acesso em: 12 out. 2017.

SILVA, W. B.; D’ALGE, J. C. L; FONSECA, L. M. G. Avaliação da qualidade geométrica de imagens da Câmera HR do CBERS 2B. In: Simpósio Brasileiro de Sensoriamento Remoto (SBSR), XIV, 2009, João Pessoa. Anais..., p. 2193-2200, Natal, 2009.

STATELLA, T. Geometric quality assessment of CBERS-4 MUXCAM image. Revista Brasileira de Geomática, v. 4, n. 2, p. 116-121, 2016.

TOUTIN, T. Geometric processing of remote sensing images: models, algorithms and methods. International journal of remote sensing, v. 25, n. 10, p. 1893-1924, 2004.

RODRIGUES, T. L.; MACHADO, M. V. A novel model for orientation of linear optical satellite images based 
in the adaptation of the orbit-attitude model. Boletim de Ciências Geodésicas, v. 25, n. 3, 2019.

\section{Biografia do autor principal}

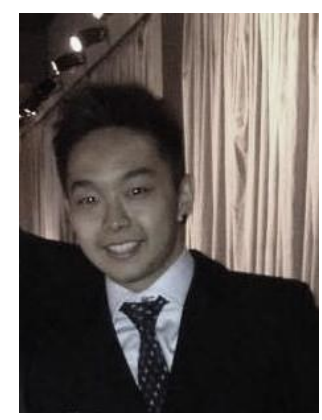

Thales Shoiti Akiyama nasceu em 1993 no município de São Paulo, SP. Possui graduação em Engenharia Cartográfica pela Universidade Estadual Paulista Júlio de Mesquita Filho - FCT UNESP, Presidente Prudente, SP. Em 2019 obteve o grau de mestre em Tecnologias Ambientais pela Universidade Federal de Mato Grosso do Sul - UFMS, Campo Grande, MS e atualmente é doutorando pela mesma universidade. É integrante do Ramo Estudantil IEEE na UFMS e do Capítulo Estudantil IEEE GRSS MS. Possui experiência na área de Geociências nas seguintes temáticas: Geomática (Cartografia, Fotogrametria, Sensoriamento Remoto, Geodésia, SIG).

Esta obra está licenciada com uma Licença Creative Commons Atribuição 4.0 Internacional - CC BY. Esta licença permite que outros distribuam, remixem, adaptem e criem a partir do seu trabalho, mesmo para fins comerciais, desde que lhe atribuam 0 devido crédito pela criação original. 